\title{
A Case Report on Treatment-Resistant Acute Exacerbation of Psychosis in Pregnancy
}

\author{
Keqing $\mathrm{Li}^{1 *}$, Morgan Pegg ${ }^{2}$ and Richard M Dreize ${ }^{3}$ \\ ${ }^{1}$ St. George's University School of Medicine, University Centre Grenada, Grenada \\ ${ }^{2}$ lbid \\ ${ }^{3}$ Department of Psychiatry, Jackson Memorial Hospital, 1611 NW 12th Ave, Miami, FL, USA
}

\begin{abstract}
Background: This case is interesting because her psychosis was exacerbated at the same time as her new pregnancy onset, and the patient had multiple gestational records. Furthermore, her psychosis was treatment-resistant to Aripiprazole.
\end{abstract}

Case Report: A 36 year-old woman with a gestational age of 27 weeks presented to the Behavioral Health Unit due to acute psychosis of carrying the "Devil's child."

Conclusion: We hope this case report can serve as a basis for establishing management guidelines for patients with peripartum acute psychosis.

Keywords: Peripartum; Psychosis; Treatment-Resistant

\section{Introduction}

Peripartum and prepartum psychosis, unlike postpartum psychosis, is a rare psychiatric emergency without systematic studies. Consequently, the management of treatment-resistant psychosis is a bit of a somewhat unchartered domain for clinicians [1]. The few peripartum psychosis management tools were based primarily on case reports of patients with bipolar disorder [2]. This case report is vital for clinicians to establish and explore treatment options for peripartum psychosis in patients with schizophrenia. We performed this case report in a community inpatient unit, and the patient verbally consented to this study.

\section{OPEN ACCESS}

${ }^{*}$ Correspondence:

Keqing Li, St. George's University School of Medicine, University Centre

Grenada, Grenada,

E-mail: kli@sgu.edu

Received Date: 01 Nov 2021

Accepted Date: 10 Nov 2021

Published Date: 22 Nov 2021

Citation:

Li K, Pegg M, Dreize RM. A Case Report on Treatment-Resistant Acute Exacerbation of Psychosis in Pregnancy. Ann Psychiatr Clin Neurosci. 2021; 4(1): 1041.

Copyright $\odot 2021$ Keqing Li. This is an open access article distributed under

the Creative Commons Attribution

License, which permits unrestricted use, distribution, and reproduction in any medium, provided the original work is properly cited.

\section{Case Presentation}

We present a G6P7 36 years-old homeless Haitian woman who was admitted involuntarily by her therapist complaining of carrying the "Devil's child." An inpatient transabdominal ultrasound did confirm that the patient is indeed 27 weeks pregnant, with no prenatal care on admission. During this episode, the patient changes between denial of the pregnancy and fixation of persecutory Haitian spirits.

The patient started having persecutory auditory hallucinations during the onset of her first trimester after she traveled back to Haiti. She met a man in Haiti's nightclub and was pregnant with him. The patient now refers to the child's father as "the Devil" and claims he tried to persecute her. The patient utterly fixated on beliefs surrounding Haitian Voodoo culture and claims that she hears seven Creole-speaking Haitians following and berating her constantly. When asked about the seven Haitians, the patient says, "they hide in my water, hide in my body, they give me poo in my hair, everywhere I go they berate me." More so, she has mentioned that "They give me food that turns me into a Jean Zombie." The patient described a long history of hearing two English-speaking American women talking, "He dead, she dead, you messed up," started in 2020 and disappeared before the Haitian voices appeared. The patient's new-onset hallucinations center around Haitian Voodoo culture and have increased intensity and frequency compared to her previous auditory hallucinations. The patient remained alert and oriented to person, place, time, and self. She maintained disorganized thought processes and intact memory, despite persistent persecutory auditory hallucinations.

The patient remained hemodynamically stable during the hospital stay. The fetal heart rate tracing performed by the hospitalist care team measured fetal heart rate at roughly $150 \mathrm{bpm}$ during hospitalization. The patient's CBC, CMP results were unremarkable. Her urinalysis was 
unremarkable except for RBC of 62. The patient's past psychiatric history is significant for psychosis and depression with multiple prior Baker Acts. Her past medical history is insignificant, except for wisdom tooth extraction, for which we do not know the exact date. The patient had six-term pregnancies in 2003, 2005, 2012, 2015, 2016, and 2017. She tested negative for STIs, including C. trachomatis, $N$. gonorrhea, Hepatitis B, Hepatitis C, Rubella, Syphilis (Treponema pallidum), and Varicella. She is currently homeless and unemployed, with no reported family locally. She was born in Haiti and immigrated to the United States in 2009 when she married a U.S. citizen. This ex-husband died from cancer in 2012, and she subsequently became impregnated by someone else. Her subsequent pregnancies were with multiple men, and all of her children were either adopted or taken in by an ex-partner's care. The patient denies the use of illicit substances and tobacco. She endorses drinking socially.

Based on collateral from the patient's ex-partner, her first auditory hallucination started about three years ago during one of her pregnancies. It persisted despite being on a therapeutic dose of Aripiprazole daily. The onset of her current pregnancy exacerbated her psychosis and depression. Inpatient psychotropic management started with Aripiprazole $5 \mathrm{mg}$ every night before bedtime, gradually increasing to $15 \mathrm{mg}$ daily without improving her persecutory auditory hallucinations.

\section{Discussion}

During pregnancy, the human brain goes through structural and biochemical changes. The pituitary gland increases in size [3], gray matter reduces in regions serving social cognitions [4], FSH becomes undetectable, and prolactin, estrogen, progesterone, and oxytocin increase. Gray matter reduction in the temporal areas is also a consistent finding in schizophrenia [5], which coincides with the areas affected by pregnancy. This case was unique because the patient had long-established schizophrenia initially believed to be triggered by pregnancy, which persisted and worsened during her multiple pregnancies. Her homelessness and medication non-compliance are also critical psychosocial factors that account for her recent psychotic episode [6,7]. Furthermore, it's essential to acknowledge that Haitian beliefs play a crucial part in psychosis that may be culturally acceptable in Haiti $[8,9]$. Despite increasing Aripiprazole from $5 \mathrm{mg}$ per day to $15 \mathrm{mg}$ per day, the psychosis persisted despite her mood improvement. In addition, long-acting Aripiprazole was avoided for unknown impacts on pregnancy [10]. This case warrants further exploration of pathogenesis in the potential deterioration of psychosis associated with pregnancy.

\section{Conclusion}

Medication management of psychosis exacerbation may be insufficient in patients with long-established schizophrenia during pregnancy. Can physiological changes in the brain during pregnancy induce exacerbation of psychosis and long-term decline in high-risk patients? Further research is needed to understand the underlying pathophysiology and develop management accordingly.

\section{Acknowledgment}

This study was supported by Dr. Richard M Dreize at Jackson Memorial Hospital.

\section{References}

1. Munk-Olsen T, Maegbaek ML, Johannsen BM, Liu X, Howard LM, di Florio A, et al. Perinatal psychiatric episodes: A population-based study on treatment incidence and prevalence. Nature Publishing Group; 2016.

2. Benjamin BD, Gordon-Elliott JS, Chaudhry SK. The Cornell peripartum psychosis management tool: A case series and template. Psychosomatics. 2016;57(3):319-24.

3. Soma-Pillay P, Nelson-Piercy C, Tolppanen H, Mebazaa A. Physiological changes in pregnancy. Cardiovasc J Afr. 2016;27(2):89-94.

4. Hoekzema E, Barba-Müller E, Pozzobon C, Picado M, Lucco F, GarcíaGarcía D, et al. Pregnancy leads to long-lasting changes in human brain structure. Nature Publishing Group. 2016.

5. Karlsgodt KH, Sun D, Cannon TD. Structural and functional brain abnormalities in schizophrenia. Curr Dir Psychol Sci. 2010;19(4):226-31.

6. Ayano G, Tesfaw G, Shumet S. The prevalence of schizophrenia and other psychotic disorders among homeless people: A systematic review and meta-analysis. BMC Psychiatry. 2019.

7. Qureshi ZP, Khandker R, Shepherd J, Samant S, Chekani F, Bailey HML. Assessment of real-life outcomes in schizophrenia patients according to compliance. Psychiatry J. Hindawi. 2020.

8. Auguste E, Rasmussen A. Vodou's role in Haitian Mental Health. Glob Ment Health (Camb). 2019;6:e25.

9. Desrosiers A, Fleurose SS. Treating Haitian patients: Key cultural aspects. Am J Psychother. 2002;56(4):508-21.

10. Iqbal MM, Aneja A, Rahman A, Megna J, Freemont W, Shiplo M, et al. The potential risks of commonly prescribed antipsychotics: During pregnancy and lactation. Psychiatry. 2005;2(8):36-44. 